\title{
DISCAPACIDAD COMO CONSTRUCCIÓN SOCIAL EN FRANCIA Y URUGUAY.
}

\author{
HANDICAP AS A SOCIAL CONSTRUCTION IN FRANCE AND URUGUAY.
}

\section{María Noel Míguez P. ${ }^{1}$}

\begin{abstract}
Resumen
A partir de un encuentro con el Prof. Henri-Jacques Stiker, uno de los autores de mayor relevancia en la temática de la discapacidad en Francia, se plantea la realización de un análisis reflexivo sobre algunas cuestiones con él conversadas.

El devenir del concepto de discapacidad ("handicap") marca la diferencia ya en el punto de partida entre Francia y Uruguay. Éste se torna clave en la forma de nombrar una temática que, quizá luego en sus contenidos, halle similitudes en el andar. Por ello se delimitarán aspectos en su distinción tales como: discapacidad y deficiencia, integración e inclusión, modelo médico ("individual") y modelo social, la cuestión de la normalidad, así como los marcos normativos y las clasificaciones nacionales e internacionales. Ese camino es el que se intenta deconstruir en el presente artículo, de manera de generar un insumo teórico que dé cuenta de las similitudes y diferencias en la conceptualización, puntos de partida y devenir de la discapacidad en Francia y Uruguay.
\end{abstract}

\section{Palabras claves:}

Discapacidad; Ideología; Normalidad; Igualdad

\begin{abstract}
:
From a meeting with Prof. Henri-Jacques Stiker, one of the most important authors on the subject of handicap in France, it is done an article containing the issues analysed with him trough the main topics about handicap.

The evolution of the concept of disability ("handicap") makes a difference from the beginning between France and Uruguay. This turns a key in naming a topic that, perhaps, in its contents could have more similarities than one can imagine. Therefore aspects, defined on its distinction such as disability and impairment, integration and inclusion, ("individual") medical model and social model, the issue of normality and regulatory frameworks, and national and international classifications.

That is the way that tries to deconstruct this article, in order to generate a theoretical input to account for the similarities and differences in the conceptualization, starting points and future of handicap in France and Uruguay.
\end{abstract}

\section{Key words:}

Handicap; Ideology; Normality; Equality

1 Docente/Investigadora de la Facultad de Ciencias Sociales de la Universidad de la República (Uruguay). Posdoctora en Prácticas y Representaciones Políticas de la Universidad de París 7 - Denis Diderot (Francia); Doctora en Ciencias Sociales de la Universidad de Buenos Aires (Argentina); Magister en Servicio Social de la Universidade Federal do Río de Janeiro (Brasil); Licenciada en Trabajo Social de la Facultad de Ciencias Sociales de la Universidad de la República (Uruguay). Coordinadora de Grado de la Lic. en Trabajo Social del Departamento de Trabajo Social de la Facultad de Ciencias Sociales y de la Regional Norte. Mail de contacto: marianoel.miguez@cienciassociales.edu.uy Dirección postal: Facultad de Ciencias Sociales, Constituyente 1502, Piso 4, Montevideo, CP 11200, Uruguay. Teléfonos: 0059824136397 


\section{INTRODUCCIÓN}

El presente artículo se titula "Discapacidad como construcción social en Francia y Uruguay", ya que más allá que los puntos de partida y devenir en la deconstrucción del concepto de discapacidad resultan bastante disímiles entre estos países, subyace la recurrencia de la (des)igualdad como concepto predeterminante para la construcción de la discapacidad en lo social. (Des)igualdad como igualdad discursiva/desigualdad inmanente y fáctica. Igualdad como concepto poco nombrado explícitamente pero sí persistente en el discurso implícito en cuanto transversalidad de la discapacidad como categoría analítica, tanto en el plano del pensar (el pensamiento abstracto de quien se objetiva investigando), y en los procesos de concreción de la misma, en el plano del ser (la realidad concreta).

En este sentido, se intenta dar cuenta del surgimiento del concepto de discapacidad ("handicap", en el francés), su devenir y sus implicancias, tanto en Uruguay como en Francia, transitando un camino que se recorre de la mano de Henri-Jacques Stiker, uno de los pensadores franceses de mayor relevancia en torno a la temática. Se toman los ejes centrales conversados con él en una entrevista que se mantuvo en el mes de enero de 2013, en París, y de ahí su deconstrucción analítica a partir del proceso de esta temática en ambos países.

Se entiende que en un proceso de investigación, la delimitación del objeto se va fundando sobre "lo ya conocido", en una díada constante entre lenguaje y pensamiento, entre ilusiones gramaticales que surgen del proceso singular de entendimiento y el discernimiento de la realidad. Ser y pensar conjúganse así como un proceso donde el investigador queda imbuido y embestido por saberes ajenos y propios, por percepciones y sensaciones, por conocimiento científico acumulado y especulación pragmática de una vida vivida en un tiempo y espacio concreto.

Así, al decir de Wittgenstein, la representación sinóptica implica una significación fundamental, puesto que designa la forma de representación de cada singularidad en tanto la forma en que se ven las cosas. Por ello, alega, "la claridad a la que aspiramos es en verdad completa". (1998, p. 255) En este sentido, "el pensamiento contiene la posibilidad del estado de cosas que piensa. Lo que es pensable es también posible". (Wittgenstein, 1979, p. 49)

Tomando estos fundamentos teóricos y epistemológicos como base, se apunta a una lógica de exposición del presente artículo en el cual es preciso dejar en claro que: "... las formas de entender y proponer las alternativas cognoscitivas son diversas; y por ello cada uno de los proponentes expone y defiende sus argumentos para exaltar las virtudes de sus teorías cognoscitivas y ponderar las bases metodológicas que de ahí se desprenden". (Massé Narváez, 2003, p. 2) Así, en encuentros de saberes en torno a temáticas que implican como punto de partida la distinción entre nosotros-otros en la relación sujeto-objeto, la deconstrucción que se haga de los conceptos irá acompañada por aspectos éticos, políticos, subjetivos, contextuales y situacionales de cada sujeto cognoscente. La potencialidad de ello se encuentra en la conjunción de saberes como forma de objetivación y deconstrucción de la temática, no en la prevalencia de un saber sobre otro.

Por ello, en este recorrido junto al Prof. Stiker, resuenan las palabras de Sartre, en tanto "el descubrimiento de mi intimidad me descubre al mismo tiempo el otro, como una libertad colocada frente a mí". (1947, p. 41)

\section{Handicap en su concepción y construcción social de la discapacidad.}

La palabra handicap ${ }^{2}$ fue introducida para contribuir a una igualdad de oportunidades entre las

2 La palabra handicap es traducida al español como discapacidad. Como se verá, por el peso en el significado etimológico de handicap resulta el primer punto de distinción en la forma de nombrar y el contenido implícito en ello. Esta palabra es introducida en la Academia Francesa en 1913 (Harmonet, Jouvencel, 2013), tomando en cuenta el concepto inglés handicap, y no siendo traducida para evitar la desaparición de su contenido real. 
personas, en este caso, aquellas que parten de condiciones de base diferentes. Quien primero menciona la palabra handicap es Samuel Pepys, en 1660, para referirse a una práctica particular. Se trataba de un método de intercambio de objetos personales de distinto valor entre dos individuos, bajo la mirada de un árbitro que era el encargado de codificar la diferencia del valor de los dos objetos y a partir de allí hacer partes iguales. La suma correspondiente era depositada en un gorro ("hand in $\mathrm{cap}^{\text {"3) }}$ ), accesorio que da nombre a la palabra handicap. (Harmonet y Jouvencel, 2013)

Posteriormente, esta concepción es incluida en las carreras de caballos para dar a todos los corredores la misma igualdad de posibilidades, siendo el punto de partida desigual por el lugar ocupado en la pista y la forma de ésta. La palabra comienza a ser utilizada en la vida cotidiana atribuyéndole un sentido general para todas las situaciones de la vida que podían ubicar a un individuo en dificultades con relación a los otros. En todo su sentido etimológico, la palabra handicap tiene en su concepción la igualdad de oportunidades.

Stiker plantea que la palabra handicap aparece a mediados del siglo XX para designar una situación en la cual estaban incluidas, por un lado, las personas que eran Ilamadas infirmes ${ }^{4}$ hasta ese momento de la historia, y, por el otro, a los heridos a partir de las dos guerras mundiales en las que Francia tuvo parte activa durante la primera mitad del siglo XX y que quedaban con secuelas, fundamentalmente evidenciadas para la imposibilidad de retornar al trabajo. Había que encontrar una nueva manera de mirar y concebir a esta población, por lo que se decide tomar de las carreras

3 "la mano en el gorro"

4 En francés se plantea el término infirmité, que de alguna manera sería el posteriormente y construcción social mediante incluido como término de deficiencia. Sin embargo, en Francia específicamente se distingue infirmité de déficience, planteándose su correlato en el español más, pudiera ser, en la palabra invalidez asociada a la imposibilidad de realizar tareas de la vida cotidiana, no así a la carga negativa que en el español contiene dicho término. de caballos la palabra handicap para comenzar a desandar su conceptualización.

Con el concepto handicap, y pensando fundamentalmente con relación a los heridos de guerra y a quienes habían tenido accidentes laborales con secuelas, se plantea desterrar la noción pura de infirmité, de falta, para significar que la persona era susceptible de encontrar un espacio en la vida social más allá de su diferencia.

Para Stiker, así como muchos autores europeos y algunos latinoamericanos que dan batalla para ello, resulta evidente que la discapacidad ${ }^{5}$ es una construcción social, ya no sólo en su etimología, sino en su conceptualización. Cierto es que hay una realidad biológica, orgánica, una marca corporal, pero todo lo demás es una construcción social. En este sentido, existen varios autores rioplatenses que hoy discuten también la condición de construcción social de los conceptos de deficiencia como marca corporal, vinculándolo a la dualidad normalidad/anormalidad con la transversalización ideológica como telón de fondo. (Rosato y Angelino, 2009; Míguez, 2009)

En los procesos societales, los contenidos y las formas de nombrar hacen al ubicarse dentro de los distintos temas que las convocan en su tiempo y espacio. Con relación a la discapacidad, si se toman en cuenta las sociedades antiguas, aqueIlas que eran religiosas encontraban en la infirmité una funcionalidad en torno a los mensajes enviados por la divinidad. La construcción social de la discapacidad era una construcción a partir de una creencia en la vida religiosa, y lo que había que decodificar era el mensaje enviado por la divinidad. Cuando nacía un niño con alguna malformación, la cuestión no era saber qué hacer con ese niño sino cual era el mensaje enviado por la divinidad a través de ese niño.

5 De aquí en más se introduce la palabra discapacidad para otorgarle coherencia gramatical al texto. De todas maneras, es importante no perder de vista que handicap es el término que se deconstruye desde la literatura francesa, y en la singularidad de la entrevista mantenida con Stiker. Handicap con el contenido etimológico y conceptual explicitado, lo que dista enormemente de dis-capacidad, o sea, falta de capacidad del español. 
Que hoy día se referencia a la discapacidad en una interacción entre los factores personales, los factores contextuales, etc., es parte de un proceso histórico que ubica a este concepto de esta manera en el hoy día. Se lo comprende desde el modelo social, al menos desde los discursos, a partir de diversos consensos normativamente materializados. Pero es igualmente una construcción social que en su devenir será modificada.

En el contexto de las deficiencias y discapacidad, en cuanto a la infirmité y handicap, éstas envían directamente a la imagen que se tiene de uno mismo: "Porque es bien la imagen de lo que no quiero ser, de lo que me da miedo. No quiero tener una pierna cortada, no es deseable" 6 . Aparecen aspectos más profundos de la esencialidad humana al encontrarse con otros en la alteridad que ponen en jaque la normalidad de uno mismo. La deficiencia aparece así como una marca corporal de la cual no se quiere tener en uno mismo. Es la imagen de uno mismo enviada al otro y el espejo de ese otro que devuelve con su sustancia corporal cuestionando. La discapacidad toca profundamente la imagen de uno mismo, "...es un espejo roto, como diría Freud. Un espejo, pero roto" $"$.

En este contexto de la discapacidad como construcción social, de uno mismo (nosotros) y otro (otros), aparece la distinción entre los conceptos de inclusión e integración. Para Stiker, y con quien se comparte, la integración es el movimiento que hace una persona adaptándose a la estructura para que luego la estructura se adapte a ella; esto en la singularidad de cada caso. La inclusión introduce una idea más fuerte: es la estructura que debe prever y estar organizada de

6 "Parce que c'est bien l'image de ce que je ne veux pas être, jusqu'au j'ai peur de. Je n'ai pas envie d'une jambe coupée, ce n'est pas désirable." (Entrevista realizada a Henri-Jacques Stiker en París en enero de 2013)

7 "...c'est une miroir brisé, comment dit Freud. Une miroir, mais brisé." (Entrevista realizada a HenriJacques Stiker en París en enero de 2013) tal manera que pueda ser receptiva de las personas en situación de discapacidad. En la inclusión está primera la transformación de la estructura, de la sociedad; en la integración es la persona que debe hacer los movimientos para encontrar su lugar. La inclusión es una perspectiva de transformación del entorno.

De esta manera, podría decirse que la inclusión está más cercana al modelo social de la discapacidad y la integración al modelo médico. Los movimientos por la integración han estado siempre como una primera parte hacia la transformación del medio. Por ejemplo, cuando se plantea el tema de la integración profesional, se dice que hay una persona en situación de discapacidad y que se debe adaptar el puesto de trabajo para esta persona, cuando, si estuvieran los puestos adaptados para todos no habría por qué distinguir tipos, lugares y formas de trabajo para unos y otros, sino que serían para todos. En el caso de la inclusión, los dispositivos para todos aparecen desde el comienzo, no están dados para individuos singulares por su situación concreta.

En las sociedades actuales, éstas más incluidas o más integradas, dependiendo de las políticas sociales, los marcos normativos, las estructuras, las sensibilidades individuales y colectivas, etc., integración e inclusión se confluyen hoy día según situaciones diversas. Ni en la sociedad más inclusiva podría decirse que el sujeto ubicado en la alteridad no hace algún esfuerzo para adaptarse; así como en la más integradora no aparecen espacios de contemplación desde lo colectivo hacia lo singular.

\section{Normalidad e ideología de la normalidad}

La normalidad aparece en este contexto, también, como una construcción social. ¿Qué es si no la normalidad? Es una cierta manera de ser, es una cierta medida promedio para poder estar. Media para ser y estar en las cuestiones cotidianas de la vida: las sillas de un tamaño estándar, las mesas, los sanitarios, etc. Es una estandarización. Pero eso no significa que sea una normalidad. 
Stiker plantea que no es más normal alguien que está dentro de la media estandarizada de alguien que no lo está, lo que da un giro interesante a las discusiones en torno a la normalidad. Ésta tomada como la media, como promedio, sin carga valorativa condicionante de los sujetos en su singularidad, sino una manera de apropiarse de los espacios de la sociedad para vivir en ella. Es una referencia obligada. En lo teórico aparece la normalidad vista como un ideal y ahí el punto de quiebre.

"Todo el mundo es normal, no hay ser viviente que no sea normal"s, plantea Stiker en la entrevista realizada en enero de este año en París. Y, agrega:

"Todo el mundo tiene normas. Entonces, las normas de las personas de talla pequeña son pequeñas. Todos los seres vivos tienen normas. Entonces, cuando es definida una normalidad, es definida una media que puede tener una utilidad práctica. Hay que encontrar un sistema de diseño para que todos puedan utilizar las cosas". ${ }^{9}$

El problema es cuando se confunde la normalidad como la media, que es una medida de utilidad para la vida cotidiana en tanto la mayoría de las personas utilizan esa media en el objeto o uso. Pero hay que hacer que este dispositivo sea lo más utilizable posible por todos, incluyendo los que no entran dentro de esa media y que quieren hacer uso de los mismos.

8 "Tout le monde est normal, il n'y a pas d'être vivant qu'il ne soit pas normal." (Entrevista realizada a Henri-Jacques Stiker en París en enero de 2013)

9 "Tout le monde a des normes. Alors, des normes des personnes de petit taille c'est d'être petit. Tous les êtres vivant ont des normes. Donc, quand est défini une normalité, on est défini une moyen que peut avoir une utilité pratique. Il faut peut-être qu'on trouve un système d'aménagement pour que tous puissent utiliser les choses." (Entrevista realizada a Henri-Jacques Stiker en París en enero de 2013)
De alguna manera, se podría retomar este planteamiento de Stiker y delimitar la normalidad como ideología en el plano del pensar y habilitarse a repensar la normalidad como circunstancia de apropiación de los usos de la vida cotidiana en el plano del ser, donde la media aparece como una referencia, como un punto de equilibrio, el cual hoy día puede ser uno y en otro momento otro. La dinámica en el concepto de normalidad puede encontrar un potencial interesante a continuar delimitando para retomar de este concepto lo reivindicativo y salir de la categórica distancia por la carga negativa atribuida.

En el plano del ser, de las representaciones caóticas, en el plano del entendimiento puro e intuitivo, normalidad se asocia directamente con la media y, contrariamente, aquel que no encuentre su espacio en la media no sería normal. Según Stiker, espontáneamente los individuos y los grupos sociales, de todas las clases y culturas, piensan que ellos son la "buena referencia", que ellos son la normalidad. Y así juzgan a los otros como los diferentes, como los que no están dentro del ideal. En sus palabras, "hay un prejuicio por las personas que son diferentes de mi, que son diferentes del grupo. La normalidad es justamente pensada como la forma correcta". ${ }^{10}$

Reflexionando en torno a la normalidad y la evidencia de una ideología de la normalidad que dé cuenta de estas cuestiones, Stiker platea que, efectivamente, todo es ideología. Ésta como un espacio irreductible y profundo de la psiquis humana. En este sentido, se cree en la ideología como tal en el plano del pensamiento, en el intercambio entre sujetos autoconscientes, como aspecto para leer el plano del ser, la realidad concreta, con los lentes que permitan la desnaturalización. Así, como se había planteado, la normalidad en el plano del pensar adquiere una irreductible cuestión

10 "ll a un préjudice pour les gens qui sont différentes de moi, qui sont différents du groupe. La normalité est justemente pensée comment la bonne forme." (Entrevista realizada a Henri-Jacques Stiker en París en enero de 2013) 
de disputa, de disrupción; en el plano del ser, se concretiza en formas de hacer de la vida cotidiana un espacio a ser usado, sentido, reproducido por todos, cada uno desde su singular normalidad.

Pero cual boomerang, se vuelven a mezclar plano del ser y plano del pensar entre normalidad, estandarización, deficiencia, otredad... El otro, en cualquiera de los planos, es atravesado por la ideología de la normalidad, desde lo más abstracto a lo más concreto, más allá se siga reivindicando la potencialidad en el cambio de figura del contenido del concepto de normalidad. Stiker lo plantea claramente, cuando da el ejemplo de qué sucede si se le pregunta espontáneamente a alguien en la calle qué opina sobre alguien que tiene una deficiencia motora, o problemas en la marcha, o que no puede hablar, o ver, etc. Intuitivamente va a pensar que no está bien, que no es "normal"; normal de estandarización, pero fundamentalmente normal de concepción de cuerpos, de marcas y presencias corporales.

Se entiende que esto se debe a las formas de interiorización y exteriorización (Sartre, 1947) de los aspectos de la vida cotidiana y las formas en las que se producen y reproducen. Si desde niños se aprende que la norma estandarizada responde a un ideal de cómo deber ser, se va a producir y reproducir esa interiorización como verdad exteriorizada. Así, la normalidad será lo positivo de la anormalidad que encontrará su negativo, en tantos pares dialécticos en pujas y contradicción.

En este sentido, Stiker plantea que todas las sociedades interiorizan su propia manera de vivir y llevar adelante sus normas. Todas las sociedades, sin excepción de una, lo hacen. Por ello hay que hacer un esfuerzo intelectual para salir de este marco. Es necesario comprender que hay unos y otros, lo cual no implica superioridad o inferioridad, sino distinciones en las formas de dar cuenta de sus normas, de su vivir en sociedad. Y eso es lo que resulta más difícil a las personas. Con estas ideas, se considera que se habilita a entender la alteridad como cercanía y no como distancia, como potencialidad y no como distorsión.
De todas maneras, continúan habiendo prejuicios. No hay que hacerse muchas ilusiones hoy día con cambios tan radicales. Hay que estar continuamente ampliando los horizontes de las personas, en sus formas de pensar y pensar(se) y en sus maneras de estar en sociedad. Las culturas de hoy día han progresado con relación al tema de la discapacidad, fundamentalmente por el tema de la mundialización en tanto espacios sociales distintos que interpelan las nociones de cada sociedad en torno a la temática, pero aún falta mucho camino por recorrer.

\section{Formas de nombrar, clasificar y normativizar}

Para comenzar, en este último punto del trabajo se traen a colación cuestiones planteadas por Stiker en algunos de sus textos. En el tema de las clasificaciones y calificaciones, en torno a la discapacidad, las designaciones espontáneas dan cuenta de las clasificaciones implícitas. De ahí la importancia que se le atribuye a las formas de nombrar.

Como se planteó, la forma de nombrar handicap contiene el concepto potencial de igualdad; no así discapacidad que incorpora negativamente (prefijo dis) las capacidades que una persona no tendría según lo estipulado por quien sí se atribuye el tenerlas. Handicap, término anglosajón e incorporado en la lengua francesa, en su forma de nombrar, etimología y devenir, se refiere al contexto; discapacidad, del español, hace ahínco en la persona. Estas formas de nombrar, ideología mediante, marca las producciones y reproducciones de los marcos normativos de uno y otro país (Artículo 2 de la Ley del año 2005 para Francia; Artículo 2 de la Ley 18.651 para Uruguay).

Desde la clasificación planteada por Esquirol, propia de la epistemología del siglo XIX, comienzan a demarcarse los individuos a corregir, en principio por educadores y médicos. Se muestra en esta clasificación inicial cómo las instituciones (salud y educación, en este caso concreto), piensan a las personas con alguna deficiencia en tanto 
poblaciones a gestionar, como riesgos a prevenir, como individuos a satisfacer. El individuo es así visualizado en una extranjería casi irrepresentable. (Stiker, 2009)

Tal como se planteó en puntos anteriores, las personas en situación de discapacidad son ubicadas en la alteridad, y así clasificadas y calificadas por sus características entendidas como diferentes a la norma. Claro es que las personas en situación de discapacidad no conforman un grupo homogéneo, sino que en esa alteridad atribuida se incorporan tantas deficiencias y sus especificidades como la ciencia se ha dado el gusto de atribuir desde su saber/poder y el derecho de dejarlas plasmadas en sus marcos normativos.

El primer documento que en estas condiciones apareció fue la Clasificación Internacional de Deficiencias, Discapacidades y Minusvalías (CIDDM), de la Organización Mundial de la Salud (OMS), en el año 1980. Aquí se incluían tres grandes tipos de especificidades: las Deficiencias/ Déficiences (visual, motriz, mental, auditiva, etc.), las Discapacidades/Invalidités (comportamiento, locomoción, movilidad del cuerpo, etc.), las Minusvalías/Désavantages (tener un rol social). Nuevamente aparece una distinción en las formas de nombras entre el francés y el español que dan cuenta de los contenidos, fundamentalmente entre minusvalías (valer menos, carga valorativa atribuida por el nosotros) y désavantages (desventajas, atribuidas al entorno directamente, no a los sujetos en su esencialidad). De todas maneras, el espíritu de esta clasificación lleva a los individuos en situación de discapacidad a ser aprehendidos como una población que presenta diferencias con la norma estipulada para el funcionamiento común. Cada individuo es comparado con relación a estándares establecidos; los sistemas de uso cotidiano son fabricados para un ciudadano promedio. Según Stiker, "c'est le moment où la santé prend un sens étendu". (2009, p. 468) La CIDDM se introduce de lleno en la salud pública, la que pone el acento en la prevención y progresivamente en los factores de riesgo, utilizando estadísticas que permitan identificar tales factores susceptibles de favorecer la aparición de enfermedades. En este contexto, la discapacidad va a estar mediada por instituciones destinadas a reeducar y reinsertar a las personas con "deficiencias" en los distintos espacios sociales.

En el año 2001, la OMS hace una segunda publicación superadora de la anterior, esta vez bajo el rótulo de Clasificación Internacional sobre el Funcionamiento (CIF). Ya no se refiere más a "consecuencias de las enfermedades", sino a "componentes de la salud". La salud es definida por los componentes que la determinan por lo que las consecuencias se focalizan en el impacto de la enfermedad o de todo problema de salud que pueda generar. Determina, a su vez, tres dominios donde el ser humano las puede tener: su cuerpo, su actividad, su participación social. (CIF, 2001)

Según el análisis de Stiker (2009), que se comparte, la insistencia tan fuerte de la CIF sobre el contexto determinante hacen parecer a la CIDDM y la CIF como opuestas, la primera dando cuenta de un modelo médico de la discapacidad, la segunda de un modelo social de la discapacidad. Sin embargo, los dos documentos no difieren en sus fundamentos más esenciales. En este sentido, la CIDDM, al pretender clasificar las situaciones para poder evaluar a los individuos Ileva al plano de lo médico el tema de los funcionamientos (corporal, personal, social) y de ahí deviene la clasificación en personas en situación de discapacidad (o no). La CIF, por su parte, describe los estados de salud y los estados conexos a la salud (educación, trabajo), lo que se entiende responde a una mirada por un lado funcionalista y por otro individualista. Los ítems de la CIF son elocuentes al plantear varias formulaciones en las funciones a nivel del cuerpo, la actividad y la participación. Esto demuestra que "el aspecto propiamente psíquico se halla ausente y que la CIF es fundada sobre una antropología del comportamiento, adaptativa, convencional". (Stiker, 2009, p. 468)

Stiker (2009) continua sus planteos diciendo que se introduce el tema de la discapacidad como objeto abstracto y fáctico a la vez, apareciendo así un sujeto con comportamientos medibles: "En- 
tre el individuo inclasificable y el individuo enteramente clasificable, naufraga una población que pasea entre el individuo desviado y el individuo a normalizar. Parecería haber un hilo conductor (...). En las clasificaciones internacionales, la subjetividad es reducida a una suerte de intencionalidad abstracta...". (Stiker, 2009, p. 489) Por otra parte, se insiste en declarar a los individuos sujetos de derecho, ciudadanos completos, personas capaces, pero en un marco de individuo consumidor que deberá satisfacer la salud, el bienestar, el acceso a los bienes de todos. En este sentido, las designaciones posiblemente resultan contradictorias con las demandas y los deseos de las personas en situación de discapacidad.

En el año 2006 surge desde Naciones Unidas la Convención de los Derechos de la Personas con Discapacidad (CDPD), como el tercer gran encuadre mundial en torno a la temática de la discapacidad. Sale del contexto de la OMS, focalizado y centrado en lo médico-sanitario y se orienta hacia un marco de los derechos de las Naciones Unidas.

De todas maneras, no entran dentro del mismo orden de cosas, por lo que hoy día se dan en simultáneo en la mayoría de los países occidentales. Como plantea Stiker, la CIF intentó hacer una síntesis entre el modelo social y el modelo médico (individual) de la discapacidad (lo que en español se dice, modelo bio-psico-social). Esto es: hay individuos que participan y los factores del entorno pueden ayudar, frenar, limitar, potenciar, etc. tal participación, por lo que hay que mirar las cosas a la vez del lado del individuo y a la vez del lado de los factores externos, los factores sociales, los factores del entorno. Pero, está claro, que en el fondo la CIF no elimina el modelo médico, "y hay personas que dicen que el modelo social es el modelo de base de la CIF. No hay que exagerar"11.

Sí es cierto que la CDPD no circula sobre el mismo camino, ya que se trata de un texto para

11 "Et même il y a certains qu'on a jusqu'à dire que le modèle social est encore le modèle de base de la CIF. II ne faut pas exagérer." (Entrevista realizada a Henri-Jacques Stiker en París en enero de 2013) que los derechos sean materializados. Eso es la CDPD, un texto para hacer que todos los derechos de las personas en situación de discapacidad se concreticen en los hechos. Es un instrumento para hacer que los derechos pasen a ser realmente hechos.

Teniendo presente que la CIF se refiere a cuestiones clasificatorias de los sujetos y que la CDPD se orienta a la materialización de los derechos de los mismos, pareciera que no tendría por qué haber contradicción entre una y otra. Justamente porque no se hallan en el mismo nivel. La CIF no es un texto de derechos, sino para observar, evaluar y describir la situación. La CDPD es un texto para obligar a los Estados a organizar sus derechos en torno a esta temática. La CDPD sí reposa en un modelo social, ya que insiste en la transformación de la sociedad y en la reivindicación de los derechos.

Más allá de los modelos en torno a la discapacidad de base, las implicancias, los fundamentos, etc., aparece clara la necesidad de clasificar y de evaluar a las personas. ¿Por qué sucede esto? Según Stiker pueden haber dos razones: por un lado, es importante saber hasta qué punto el contexto en el que se habita ayuda o frena la actividad de las personas; por el otro, la CIF obliga a los legisladores a tomar en consideración los factores del entorno.

De todas maneras, el modelo clasificatorio que utiliza Francia no es la CIF, sino el de Processus de Production de Handicap (PPH), que se toma de Quebec. Stiker lo considera más pertinente ya que parte de la base del hombre como ser social, antes que un individuo en un ser en sociedad. Este modelo introduce lo que llama "habitudes de vie" ("estilos de vida"), aspectos que no plantea la CIF, y que trata de todo lo que un individuo considera necesario para vivir en torno a la familia, la educación, el trabajo, la vivienda, etc. De esta manera, los factores biológicos pasan a un segundo plano con relación a los factores sociales, los que tampoco implican sólo los factores del entorno sino más contextual. Pero, el PPH es un modelo francófono utilizado en Quebec, Francia, Belgica y Suiza. 
En Uruguay, lamentablemente, ni siquiera se conoce este modelo. Recién se está dando el salto de la CIDDM a la CIF, lo cual es tomado como de avanzada y con el discurso reivindicativo, insistente y pareciera que inapelable que se trata de un modelo bio-psico-social. Se considera que lejos se está de eso y que sólo se logra reproducir la ideología de la normalidad a través de una herramienta usada fuertemente en sus aspectos más médico-hegemónicos. Se confunden casi cotidianamente deficiencia y discapacidad sin mediar siquiera la posibilidad de interpelar tal confusión conceptual. Se clasifican las deficiencias en nombre de las discapacidades, porque ya en la forma de nombrar, lo clasificado es la deficiencia con título de discapacidad.

Con relación a los marcos normativos nacionales, Stiker plantea que la Ley del año 2005 de Francia tiene un fuerte componente de igualdad de base. Que se refiera a una población específica la hace discriminatoria por efecto. En este sentido, para contar con los derechos que esta ley plantea, es necesario declararse handicapé(e), como forma de distinguir unos de otros. A partir de esto, hay movimientos de personas en situación de discapacidad que no quieren en absoluto tener una ley particularizada en ellas, ya que los efectos de ser obligados a declararse en situación de discapacidad para acceder a los beneficios de la ley les ponen la etiqueta de handicap en la espalda. En este sentido, Stiker analiza que sí se trata de una discriminación, la cual es necesaria ya que si no existiera por la sola voluntad de las personas no habría igualdad. Sería una ilusión creerlo. Sin embargo, esta ley debe incorporar los dispositivos que no agraven las desigualdades, sino que las reduzcan.

Por su parte, en Uruguay, la Ley $N^{\circ} 18.651$ del año 2010, en los aspectos recién mencionados de la ley francesa, aparecen casi las mismas cuestiones. Sí se trata de una ley específica para una población específica, cosa que no es distintivo y único como en Francia con la ley del 2005. En Uruguay existen otras leyes específicas reconocidas también bajo el marco de la "discriminación positiva". También la ley del Uruguay prevé registro de las personas en situación de discapacidad para poder acceder a los beneficios, fundamentalmente en torno a la cuota para el ingreso al trabajo (4\% de las vacantes de los empleos públicos). Fue una ley que recibió las opiniones de las diversas organizaciones de la sociedad civil, entes públicos, espacios privados, etc. que se quisieron acercar para su elaboración. Es una ley que está sancionada pero aún no reglamentada, por lo que no tiene el peso de la sanción si no se cumple. Es una ley que tiene más discusiones para su implementación en la órbita del Estado para su implementación que en las personas en situación de discapacidad que no la cuestionan. Es una ley que desde el discurso tiene como base el modelo social de la discapacidad, pero en sus magras concreciones y de la mano de la CIF como marco de apoyo clasificatorio se mantiene en amoríos con el modelo médico hegemónico de la discapacidad.

\section{REFLEXIONES FINALES}

Tal como plantea Stiker (1982), una de las condiciones para que los unos y los otros (uno mismo y otro) se hallen en conjunción, que los otros tengan su propia palabra, está dado por las imágenes que la sociedad muestra. Con relación a lo que se ha planteado de las formas de nombrar, las clasificaciones y las normalizaciones, bajo la relatividad y la historicidad de las conceptualizaciones, es necesario desandar los caminos que fijan estereotipos y reducen las subjetividades a etiquetas.

Mientras se siga distinguiendo con marcas que niegan las singularidades y ubican al otro en una "alteridad excluyente", se considera que no habrá igualdad, de la real, de la de base. La igualdad en los discursos ya está dada, ahora urge materializarla. De todas maneras, es necesario comprender el momento histórico, el espacio social y cultural, las sensibilidades, los movimientos, etc. para construir y reconstruir una nueva noción de igualdad que discursiva y fácticamente incorpore a todos los sujetos que viven en sociedad. 
Se comparte con Stiker en que existen grandes distancias entre la producción discursiva en torno a la temática de la discapacidad y la puesta en práctica de lo dicho. Esas distancias generan dolores en sujetos que subjetivamente son interpelados en su vida cotidiana por una realidad que dista de lo normativamente dispuesto.

Pero los cambios dependen, fundamentalmente, de la fuerza que tengan los movimientos sociales que se impliquen en esta temática. Cuando al comienzo del trabajo se planteaba que en Francia el concepto de handicap fue introducido a partir de superar la noción de infirmité que no contemplaba a la gran cantidad de personas con secuelas de las guerras y de accidentes laborales, se hacía referencia justamente a esto. Los procesos devienen por movimientos, y estos deben llegar desde los espacios sociales directamente implicados.

Hoy día se está ante una nueva perspectiva donde lo principal que aparece es el tema de la transformación de la sociedad, de los marcos sociales y del entorno. Cuanto más se amplíe el entorno en tanto contexto que contiene a los sujetos, más encontrará la persona la posibilidad de ser y vivir en condiciones de igualdad, tanto unos como otros.

\section{REFERENCIAS BIBLIOGRÁFICAS}

MASSÉ NARVÁEZ, C. (2003). Del Método Trascendental Kantiano a la Dialéctica de la Razón de Hegel: Un esbozo general de sus soportes epistemológicos. Santiago de Chile: Revista Cinta de Moebio. v.17, pp. 23-48.

MíGUEZ, M.N. (2009). Construcción social de la discapacidad. Montevideo: Trilce.

MOTTEZ, B. (1996). Une entreprise de dé-nomination: les avatars du vocabulaire pour désigner les sourds au XIX et XX siècles. En: STIKER, H.J, VIAL, M. Handicap et inadaptation. Fragments pour une histoire: notions et acteurs. Paris: Alter. Pp. 101-120.

ROSATO, A., ANGELINO, M.A. (2004). Discapacidad e ideología de la normalidad. Desnaturalizar el déficit. Buenos Aires: Noveduc.

SARTRE, J.P. (1947). L'imaginaire. Paris: Gallimard.

STIKER, H-J. (1982). Corps infirmes et sociétés. Paris: Aubier-Montaigne.

STIKER, H-J. (2009). Comment nommer les défiances?. Ethnologie française, 2009/3 Vol. 39, p. 463-470. DOI : 10.3917/ ethn.093.0463.

STIKER, H-J. (2010). Les métamorphoses du handicap de 1970 à nos jours. Soi-même avec les autres. Grenoble: Presses universitaires de Grenoble.

WITTGENSTEIN, L. (1979). Tractatus Lógico Philosophicus. Barcelona: Alianza.

WITTGENSTEIN, L. (1988). Investigaciones filosóficas. Barcelona: Crítica.

Fuentes documentales:

COMISIÓN NACIONAL HONORARIA DE LA DISCAPACIDAD. Apartado Legislación. http://www.cnhd.org/legislacion.htm Archivo revisado 17-02-13.

HARMONET, C., de JOUVENCEL, M. (s/d). L'expertise médicale et le handicap. Richebourg. http://claude.hamonet.free.fr/fr/art_expertise.htm Archivo revisado le 15 janvier 2013.

International classification of impairments, disabilities, and handicaps, a manual of classification relating to the consequences of diseases, ICIDIH (1980). Genève: World Health Organisation.

LEY No 18.651. (2010). Protección Integral de los Derechos de las Personas con Discapacidad. Uruguay: Poder Legislativo.

LOI N²005-102 du 11 février 2005 pour l'égalité des droits et des chances, la participation et la citoyenneté des personnes handicapées.

NU. (2006). Convención sobre los Derechos de las Personas con Discapacidad. Washington: ONU.

OMS. (2001). Classification Internationale du Fonctionnement, du handicap et de la santé (CIF). Organisation Mondiale de la Santé: Genève. 